\title{
Investigation of polyphenol synergism for reducing sugar release from white bread
}

Polyphenols are known to exhibit a number of health promoting effects such as reducing the postprandial glycaemic response. It has been proposed that polyphenols in combination, as found together naturally in foods exert synergistic health effects compared to isolated polyphenols. In the body, polyphenols undergo structural changes throughout digestion which can alter their bioavailability and thus their health impact.

Previous results from our lab found that after being baked into white bread in isolation, the polyphenol rich green tea extract (GTE) at $0.4 \%$, grape seed extract (GSE) at $0.2 \%$, resveratrol (RES) at $0 \cdot 22 \%$ and baobab fruit extract (BAO) at $1.88 \%$ reduced rapidly digestible starch (RDS) ${ }^{(1,2)}$. RDS takes place 20 minutes into the duodenal stage of digestion when sugar release is most pronounced and therefore contributes greatest to the glycaemic index (GI) of the food. Using the same optimal isolated extract doses for reducing sugar release from bread in previous studies (with a 75\% variation to increase the variety of extract combinations used), the objective was to determine if polyphenol rich extracts showed a synergistic effect in reducing sugar release from high GI white bread.

Bioaccessibility of polyphenols was analysed using an in vitro digestion model adapted from Ryan et al. ${ }^{(3)}$ to determine the stability of polyphenols throughout the digestive process. RDS sugar release was measured using the method by Mishra \& Monro ${ }^{(4)}$ followed by a colourimetric method by Englyst \& Hudson ${ }^{(5)}$.

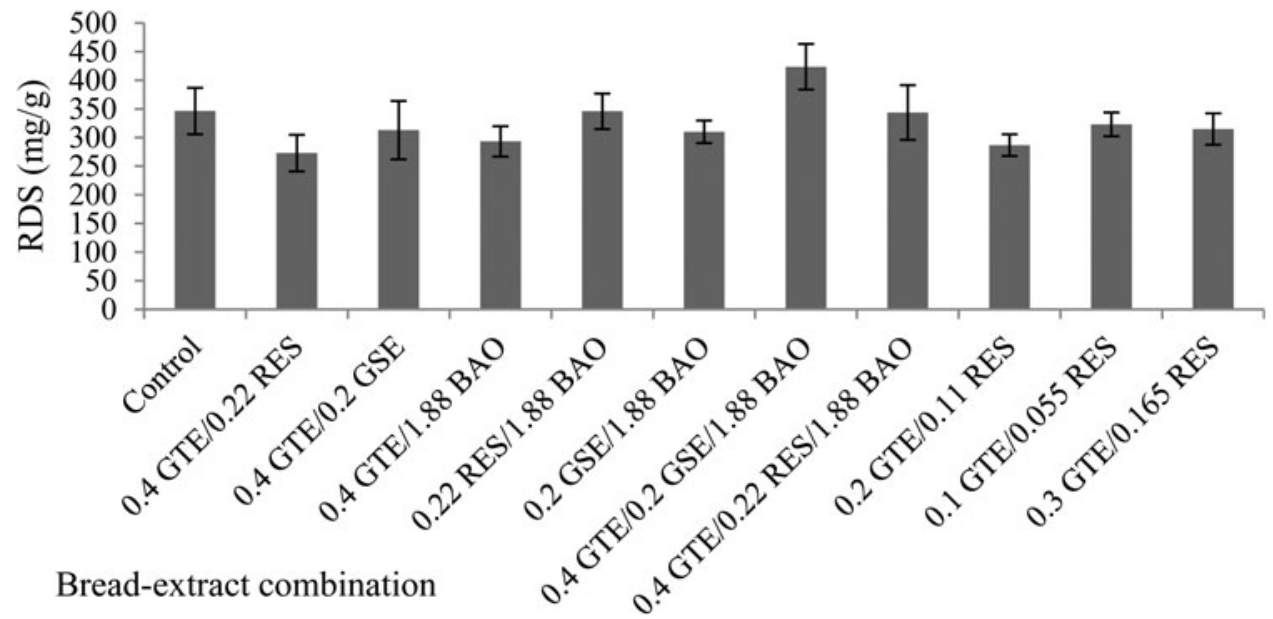

Fig. 1 Rapidly digestible starch (RDS) sugar release from white bread with combinations of polyphenol extracts. Means of 3 experiments with standard error (ANOVA, followed by Tukey test) and based on $\%$ of each extract added relative to $500 \mathrm{~g}$ loaf.

Bioaccessibility increased for all extracts $(p<0 \cdot 05$; non-significant for RES) illustrating that polyphenols become potentially more available in the body throughout digestion. Although polyphenol rich extracts in isolation were shown to reduce sugar release from high GI bread, in combination there was no reduction. Therefore, many factors must be considered when using polyphenols as functional food additives.

1. Coe S \& Ryan L (2013) Proc Nutr Soc, 72, E201.

2. Coe $\mathrm{S}$ et al. (2013) Nutr Res, 33, 888-896.

3. Ryan L et al. (2008) Pla Food Hum Nutr, 63, 127-133.

4. Mishra S \& Monro JA (2009) J Cereal Sci, 50, 61-66.

5. Englyst HN \& Hudson GJ (1987) Food Chem, 24, 63-76. 